\title{
Towards a Common Language of Infrastructure Interdependency
}

\author{
Neil Carhart ${ }^{1}$ and Ges Rosenberg ${ }^{2}$ \\ ${ }^{1}$ International Centre for Infrastructure Futures and The Systems Centre, University of Bristol, UK \\ ${ }^{2}$ The Systems Centre, University of Bristol, UK
}

\begin{abstract}
Infrastructure systems can exist interdependently with one another either by design, necessity or evolution. There is evidence that interdependencies can be the source of emergent benefits and hazards, and therefore there is value in their identification and management. Achieving this requires collaboration and communication between infrastructure stakeholders across all relevant sectors.

Recognising, developing and sharing multiple understandings of infrastructure interdependency and dependency will facilitate a wide range of multi-disciplinary and cross-sectorial work and support productive stakeholder dialogues. This paper therefore aims to initiate discussion around the nature of infrastructure interdependency and dependency in order to establish the basis of a useful, coherent and complete conceptual taxonomy. It sets out an approach for locating this taxonomy and language within a framework of commonplace stakeholder viewpoints.
\end{abstract}

The paper looks at the potential structural arrangements of infrastructure interdependencies before exploring the qualitative ways in which the relationships can be characterised. This builds on the existing body of knowledge as well as experience through case studies in developing an Interdependency Planning and Management Framework for Infrastructure.

Keywords: Interdependency, Resilience, System Architecture

\section{SUBSTITLE REQUIRED}

Infrastructure systems can exist interdependently with one another either by design, by necessity or through evolution. There is widespread evidence that interdependence can be the source of emergent benefits and hazards, and therefore there is value in its identification and management ${ }^{1}$. Achieving this requires collaboration and communication between infrastructure stakeholders across all relevant sectors. Collaboration can develop Situational Awareness, that is to say a holistic knowledge of the infrastructure landscape and therefore potential interdependencies.

As a result of the perceived vulnerabilities and opportunities which emerge from infrastructure interdependency, there is an increased interest in modelling and understanding them².

There are many methods for modelling the interdependency between infrastructure elements, each serving a

1 Frontier Economics. Systemic Risks and Opportunities in UK Infrastructure - A Report Prepared for HM Treasury \& Infrastructure UK. (2012).

2 HM Treasury \& Infrastructure UK. National Infrastructure Plan 2011. (2011). 
specific purpose and providing a different conception of what interdependency means in relation to infrastructure. Some differing conceptions of infrastructure interdependency can be attributed to the multiple viewpoints of the stakeholders responsible for commissioning, financing, planning, designing, building, operating and using infrastructure. For example, there was a significant increase in research into infrastructure interdependencies following the Oklahoma City bombing in $1995^{3}$, primarily as a result of Presidential Decision Directive $63^{4}$ which stressed the importance of infrastructure interdependency in terms of national vulnerability. This influenced a focus on the vulnerability emergent from interdependency. The resultant modelling tools focussed on understanding those risks, often as discrete from understanding the benefits of interdependency.

Without a reflection on the meaning of infrastructure interdependency, and without the means to describe it completely and consistently, there is a danger that one particular understanding of infrastructure interdependency, for example in terms of producing vulnerability; one particular modelling approach, for example network theory; or one particular type of interdependency, for example the physical transfer of resources; become dominant at the expense of others.

The complete, holistic view of a system can rarely be effectively captured by one single model or modelling approach. Instead it is necessary to have a meta-model or a framework which brings together all models which represent different aspects and views of the system into one coherent and internally consistent architecture, as advocated by Zachmann ${ }^{5}$ and Kruchten ${ }^{6}$ and used in architectural frameworks in many domains (e.g. DoDAF ${ }^{7}$ or TRAK $^{8}$ ). In formulating a ' $4+1$ ' view architecture, Kruchten shows how to represent concurrently and coherently four viewpoints on a complex software development programme, comprising 1) logical, 2) process, 3) development and 4) physical views, each represented and best-served by a different modelling approach. These are complemented by a fifth enduser view. These system architectures, in which multiple perspectives are developed for the same underlying system, have the advantage of providing an efficient and effective means of communicating with multiple stakeholders.

In a similar way, the language of infrastructure interdependency and any associated taxonomy needs to recognise the likelihood that different stakeholders will have differing perspectives on a network of infrastructure and on the associated interdependencies. The language and taxonomy therefore needs to provide a means of relating the shared understanding of infrastructure interdependencies to the interests of the different stakeholders. For example, an engineering perspective may tend to focus on physical interactions and information flows between infrastructure systems and use these to identify issues of network resilience. While, no doubt concerned by issues of resilience, an investor in infrastructure is also likely to take an interest in any opportunities to generate additional value through the exploitation of infrastructure interdependency.

Recognising, developing and sharing multiple understandings of infrastructure interdependency and dependency will facilitate a wide range of future multi-disciplinary and cross-sectorial work and support productive cross-sector stakeholder dialogues. This paper aims to initiate a discussion around the nature of infrastructure interdependency and dependency in order to establish the basis of a useful, coherent and complete conceptual taxonomy. It also sets out an approach for locating this taxonomy and language within a framework of commonplace stakeholder viewpoints.

There are many ways in which the interdependency within and between infrastructure networks can be modelled. For example, previous reviews ${ }^{9,10,11}$ have identified qualitative and semi-qualitative models including: Network and Graph Theory; topological models; Petri-nets; Input-Output models; Agent Based models; spatial and time-series analysis; matrix representations and hierarchical risk models.

Building these models can lead to a discussion of interdependency types with which to characterise the edges connecting the nodes in the system and therefore they help in establishing the beginnings of an interdependency taxonomy. If the different types of interdependency are not explicitly considered in the early stages of a project,

3 Heller, M. in Front. Eng. Reports Leading-Edge Eng. from 2001 NAE Symp. Front. Eng. 138 (National Academies Press, 2002

4 The Clinton Administration. Presidential Directive 63 (PDD-63): Policy on Critical Infrastructure Protection. (1998).

5 Zachman, J. A. A framework for information systems architecture. IBM Syst. J. 26, 276-292 (1987).

6 Kruchten, P. Architectural Blueprints - The "4+1" View Model of Software Architecture. IEEE Softw. 12, 42-50 (1995).

7 Department of Defense. Department of Defense Architecture Framework Version 2.0 (2009)

8 TRAK Steering Group.and UK Department for Trasnport. TRAK Enterprise Architecture Framework (2013)

9 Pederson, P., Dudenhoeffer, D., Hartley, S. \& Permann, M. Critical Infratructure Interdependency Modeling: A Survey of U.S. and Intemational Research. (2006).

10 Bloomfield, R., Salako, K., Wright, D., Chozos, N. \& Nobles, P. Infrastructure interdependency analysis: an introductory research review. (2009).

11 Ouyang, M. Review on modeling and simulation of interdependent critical infrastructure systems. Reliab. Eng. Syst. Saf. 121, 43-60 (2014). 
and each modelling approach specialises in a particular type of interdependency, then the choice of model can unintentionally narrow the view of what constitutes interdependency. Ultimately this can increase the risk of temptation, "if the only tool you have is a hammer, to treat everything as if it were a nail"12.

It is proposed therefore that there is a renewed need to reflect on the characteristics of interdependency; the modelling approaches which best provide insight into each, and the way in which these modelling approaches can be brought together into a coherent architecture framework which allows us to represent the totality of interdependency. The paper begins by examining the three most commonly discussed characteristics; directionality, order and typology, before proposing a wider set of characteristics with which infrastructure interdependency can be described.

\section{DIRECTIONALITY}

Infrastructure interdependency has been described as a distinctly bidirectional relationship ${ }^{13}$ and elsewhere in such a way that includes bidirectional and non-reciprocal dependency in the form of an influence from one element on another ${ }^{9,14}$. Eusgeld et al. ${ }^{15,16}$ differentiate between these as 'input' and 'mutual' interdependency types. It has been argued that these bidirectional relationships exist at the macro level, whereas dependencies are more common at the component level ${ }^{17}$.

\section{ORDER}

Discussions of the fundamental definition of infrastructure interdependency and its modelling have also led to the distinction between first, second and third order dependencies ${ }^{13,17}$ having been previously proposed as an important part of a characterisation framework ${ }^{18}$. A first order dependency is where system $A$ is directly dependent on system B, and second order dependency is where system A is indirectly dependent on system B via A's first order dependency on a third infrastructure which is itself directly dependent on B. These indirect effects can lead to feedback loops where the second order dependency means $A$ affects $B$ which then affects $A^{19}$. This implies that second order dependencies are interdependencies. Third order dependencies introduce a further intermediary system.

\section{TYPE}

There have been several attempts to characterise interdependency into several descriptive types alternatively referred to as the nature of the interdependency. One of the earliest and most frequently cited taxonomies was proposed by Rinaldi at al. ${ }^{13}$ :

- Physical (a physical output from one system is a necessary input to another)

- Cyber (information produced by a system affects the operation of another)

- Geographic (two or more systems are considered to be co-located in physical space)

- Logical (a mechanism that could be organisational or social).

These reflect four earlier interdependency typologies for product design matrices proposed by Pimmler and

12 Maslow, A. H. The Psychology of Science. (1966).

13 Rinaldi, S. M., Peerenboom, J. P. \& Kelly, T. K. Identifying, understanding, and analyzing critical infrastructure interdependencies. IEEE Control Syst. Mag. 21 , 11-25 (2001).

14 HM Treasury \& Infrastructure UK. National Infrastructure Plan 2011. (2011).

15 Eusgeld, I., Nan, C. \& Dietz, S. "System-of-systems” approach for interdependent critical infrastructures. Reliab. Eng. Syst. Saf. 96, 679-686 (2011).

16 Briš, R., Eusgeld, I., Nan, C. \& Dietz, S. "System-of-systems” approach for interdependent critical infrastructures. Reliab. Eng. Syst. Saf. 96, 679-686 (2011).

17 Johansson, J. \& Hassel, H. An approach for modelling interdependent infrastructures in the context of vulnerability analysis. Reliab. Eng. Syst. Saf. 95, 1335-1344(20).

18 MoDaniels, T., Chang, S., Peterson, K, Mkawoz, J. \& Reed, D. Empinical Frameworkfor Characterizing Infrastructure Falu re interdependencies. J. Infrastruct. Syst. 13, 175-184 (2007).

19 Little, R. G. Controlling Cascading Failure: Understanding the Vulnerabilities of Interconnected Infrastructures. J. Urban Technol. 9, 9-123 (2002). 
Eppinger $^{20}$ : Materials, Energy, Information and Spatial. Zimmerman ${ }^{2122}$, again looking specifically at infrastructure interdependency only differentiates between two types Functional and Spatial, while others ${ }^{17}$ use Functional and Geographic (where functional covers physical, cyber and logical). Pederson et al. ${ }^{9}$ use an expanded version of an earlier taxonomy ${ }^{23}$ which splits Logical interdependencies into two further groups: Policy/Procedural and Societal.

The distinction of physical, cyber and logical interdependencies is also used by Satumtira and Duenas-Osorio in

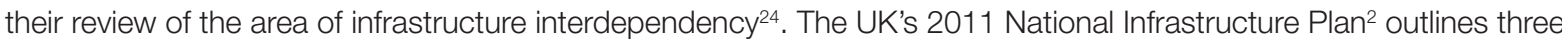
forms of interdependencies (p90):

\section{- Geographic co-location}

\section{- Shared use (of equipment or resource)}

\section{- Reliance on another network's function.}

While this aligns with the split into geographic and functional, shared use expands on the previous conception of physical interdependencies. A Frontier Economics Report ${ }^{1}$ implements a definition of interdependency proposed by $\mathrm{O}^{\prime} R o u r k e^{25}$, which suggests that it results from physical proximity or operational interaction, reflecting the two-factor split into spatial and functional interdependency. They use this to develop an economic framework within which to consider interdependency, which proposes three forms: physical, digital, and organisational (e.g. shared ownership or oversight).

The process of defining the nature of interdependency has proved to be a challenging one ${ }^{24}$. A recent analysis ${ }^{11}$ of the taxonomies of Rinaldi et al. ${ }^{13}$, Zimmerman ${ }^{21}$, Dudenhoeffer et al. ${ }^{23}$, Wallace et al. ${ }^{26}$ and Zhang and Peeta ${ }^{27}$ concluded that "some interdependency examples in practice cannot be definitely categorized by some classifications", and only the classification proposed by Rinaldi et al ${ }^{13}$ covered all ten real-world interdependency examples analysed.

While at one level of operation these dimensions may be sufficient to characterise the interdependency between two infrastructure systems, there are others that could be important, particularly in relation to specific modelling approaches. For example, the degree of coupling (from tight to loose) between systems may affect the way in which effects propagate ${ }^{19}$ invoking Perrow's ${ }^{28}$ classification of systems. Sector specific metrics can also be used such as the relative duration of a power outage in relation to the disruption it causes to the function of other systems ${ }^{22}$.

In planning and managing infrastructure interdependencies, both for resilience and efficiency, it is also necessary to consider the ways in which the interdependencies have impact and can be impacted. The case studies reported previously ${ }^{29}$ resulted in the introduction of a number of additional characteristics for describing infrastructure interdependency discussed below.

20 Pimmler, T. \& Eppinger, S. Integration analysis of product decompositions. in ASME 6th Int. Conf. Des. Theory Methodol. (1994).

21 Zimmerman, R. Social Implications of Infrastructure Network Interactions. J. Urban Technol. 8, 97-119 (2001).

22 Zimmerman, R. \& Restrepo, C. E. The next step: quantifying infrastructure interdependencies to improve security. Int. J. Crit. Infrastructures 2, (2006).

23 Dudenhoeffer, D., Permarn, M. \& Manic, M. CIMS: A Framework for Infrastructure Interdependency Modeling and Analysis. in Proc. 2006 Winter Simul. Conf. 478-485 (1EE, 2006).

24 Satumtira, G. \& Duenas-Osorio, L. in Sustain. Resilient Crit. Infrastruct. Syst. Simulation, Model. Intell. Eng. (Gopalakrishnan, K. \& Peeta, S.) 300 (Springer, 20 ).

25 O'Rourke, T. D. Critical Infrastructure, Interdependencies, and Resilience. Bridg. 37, (2007).

26 Wallace WA, Mendonça D, Lee EE, Mitchell JE, Chow JH. Managing Disruptions to Critical Interdependent Infrastructure in the Context of the 2001 World Trade Centre Attack. In: Monday JL, editor. Beyond Sept. 11th An Acc. Post-Disaster Res., (2003)

27 Zhang, P. \& Peeta, S. A generalized modeling framework to analyze interdependencies among infrastructure systems. Transp. Res. Part B Methodol. 45, 553-579 (2011).

28 Perrow, C. Normal Accidents. (Basic Books, 1984).

29 Rosenberg, G. \& Carhart, N. J. A Systems-based Approach to Creating Value from Infrastructure Interdependencies. in Int. Symp. Next Gener. Infrastruct. (2013). 


\section{FUNCTIONALITY}

It has previously mentioned that infrastructure interdependencies can be thought of as either functional or spatial|14,21,30, but it is proposed that this is an import distinction separate from the typology. In terms of vulnerability analysis, functional dependency between two elements can be of utmost importance, but in terms of opportunities and efficiencies, the creation of non-functional interdependencies can be significant. For example, the ElecLink Channel Tunnel Interconnector is said to create a geographic interdependency saving in the region of $£ 60 \mathrm{~m}$ compared to laying a seabed cable ${ }^{1}$. The Channel Tunnel Rail link does not depend on the electricity interconnector for it to function, and the interconnector does not depend on the functioning of the rail link.

\section{NECESSITY}

Strongly related to identifying whether an interdependency is based on a functional requirement is the distinction between an interdependency which already exists and is necessary or a situation where a relationship exists which is not essential to a system's operation, but in which there is the potential for the interaction to be exploited to provide additional benefits such as enhancing efficiency or resilience. There is some overlap between a necessary interdependency and a functional one, but the need to include this as a specific category arose from work looking to identify beneficial interdependency opportunities in three UK case studies ${ }^{29}$.

\section{OUTCOME}

The identified interdependencies also need to be classified as to whether they primarily offer additional opportunity to benefit or whether they result in an increase in negative risks or dis-benefit. If it is beneficial then there may be a reason to utilise an existing interdependency and leverage it for additional value or alternatively create it if there is the potential to do so. If it is hazardous, there may be a reason to mitigate or prevent the interdependency.

\section{LIFE-CYCLE IMPACT STAGE}

This refers to the chronological phase of the infrastructure's life cycle during which the impact of the interdependency is of most importance. Some interdependencies only have an impact during the Planning or Construction phase, others are important during Operation or at the infrastructure element's 'End of Life'. Additionally, some interdependencies are only relevant under particular Scenarios (such as during accidental failure or during extreme weather events). These categories also arose during the research of three UK case studies ${ }^{29}$.

\section{GEOGRAPHIC SCALE}

Identifying whether the interdependency exists on a local, regional, national or international scale may be important, as may characterising where the impact of the interdependency lies ${ }^{18}{ }^{31}$.

Table 1 compiles a checklist of interdependency categories which has been drawn from the wide range of literature describing and modelling interdependency referenced and discussed above. While all of these criteria are intended to simplify the categorisation process, there remain practical complexities. For example, assessing whether a network of infrastructure systems, taken as a whole, possess the sufficient and necessary capabilities to realise the cumulative benefits of a desirable interdependency.

A rich and shared through-life understanding of the diverse aspects of infrastructure interdependency, and an appreciation for which modelling tools best represent each of those aspects, are the first steps to creating a framework to integrate toolsets and provide a more comprehensive picture of the interdependencies in the infrastructure 'system of systems'. This has implications for the design of infrastructure assets and their integration in the wider network of a nation's infrastructure, by helping to minimise unforeseen vulnerabilities and maximise opportunity management for valuable emergent economic and social benefits that might otherwise by missed.

30 Zimmerman, R. \& Restrepo, C. E. The next step: quantifying infrastructure interdependencies to improve security. Int. J. Crit. Infrastructures 2, (2006).

31 Peerenboom, J. P. Infrastructure Interdependencies: Overview of Concepts and Terminology. in Natl. Sci. Foundation. Sci. Technol. Policy Workshop on Crit. Infrastructure. Needs in Interdisciplinary Research and. Graduate. Training. (2001). 
A review by Satumtira and Duenas-Osorio ${ }^{24}$ of 162 published papers on infrastructure interdependency modelling identified over 40 different approaches, with three in particular being much more widely used than the others.

Around $22 \%$ of the papers employed Network and Graph Theory based approaches, and around the same for Input-Output based approaches. A further \% used Agent Based Modelling, while the remaining 46\% used one or a mixture of the many other techniques. A more recent study ${ }^{11}$ highlighted how each of these techniques addresses each interdependency type, for example, they concluded that Input-Output modelling, does not effectively capture geographic or logical interdependencies.

\section{Table 1: Infrastructure interdependency characterisation checklist}

\begin{tabular}{|c|c|c|}
\hline & & $\begin{array}{l}\text { Identified } \\
\text { Interdependency }\end{array}$ \\
\hline \multirow{4}{*}{ TYPE } & Physical & \\
\hline & Digital & \\
\hline & Geographic & \\
\hline & Organisational & \\
\hline \multirow{3}{*}{ ORDER } & First Order & \\
\hline & Second Order & \\
\hline & Higher Order & \\
\hline \multirow{2}{*}{ FUNCTION } & Functional & \\
\hline & Non-Functional & \\
\hline \multirow{2}{*}{ NECESSITY } & Necessary & \\
\hline & Optional & \\
\hline \multirow{2}{*}{ OUTCOME } & Benefit & \\
\hline & Dis-benefit & \\
\hline \multirow{4}{*}{ LIFE-CYCLE IMPACT STAGE } & Planning & \\
\hline & Construction & \\
\hline & Operation & \\
\hline & Scenario & \\
\hline \multirow{4}{*}{ GEOGRAPHIC SCALE } & Project & \\
\hline & Local & \\
\hline & National & \\
\hline & International & \\
\hline
\end{tabular}

Work for Engineering the Future ${ }^{32,33}$ which investigated the interdependencies between future UK infrastructure projects and policies ${ }^{34}$ found that while $47 \%$ of the identified interdependencies could be classified as physical, $26 \%$ were logical and $13 \%$ geographic. This is not a criticism of the ability of any particular technique, but it is an argument for the need for a suite of modelling approaches, and a framework which brings them together, to truly understand all dimensions of interdependency.

In conclusion, we believe that bringing together the terminology used for the description of infrastructure interdependency, as described in this paper, is an important stage in the establishment of a common language for characterising and discussing infrastructure interdependency across infrastructure sectors and amongst different academic disciplines. It is hoped that this will initiate further discussion on the nature of infrastructure interdependency and ultimately aid in facilitating cross-sector discussions. Most importantly perhaps, it highlights the need for a framework which brings together all of the individually powerful modelling approaches in a consistent and mutually beneficial way, such that the totality of interdependency within the infrastructure system-of-systems can be made visible for the reduction of vulnerability and the exploitation of additional benefits. Without a common language with which to describe all aspects of interdependency there is a danger that an important piece of the puzzle will be overlooked.

32 The Systems Centre, University of Bristol. Workshop Application of a Matrix Based Approach to the Identification of Infrastructure Interdependencies - Workshop Report for Engineering the Future. (2013).

33 Engineering the Future. Infrastructure Interdependencies Timelines. (2013).

34 Engineering the Future. UK Infrastructure Timelines. (2011). 